Document downloaded from:

http://hdl.handle.net/10251/64211

This paper must be cited as:

Conde Castellanos, PE.; González Martínez, AJ.; Gonzalez, A.; Hernández Hernández, L.; Bellido, P.; Crespo Navarro, E.; Iborra Carreres, A.... (2015). Analysis of the Statistical Moments of the Scintillation Light Distribution With dSiPMs. IEEE Transactions on Nuclear Science. 62(5):1981-1988. doi:10.1109/TNS.2015.2473695.

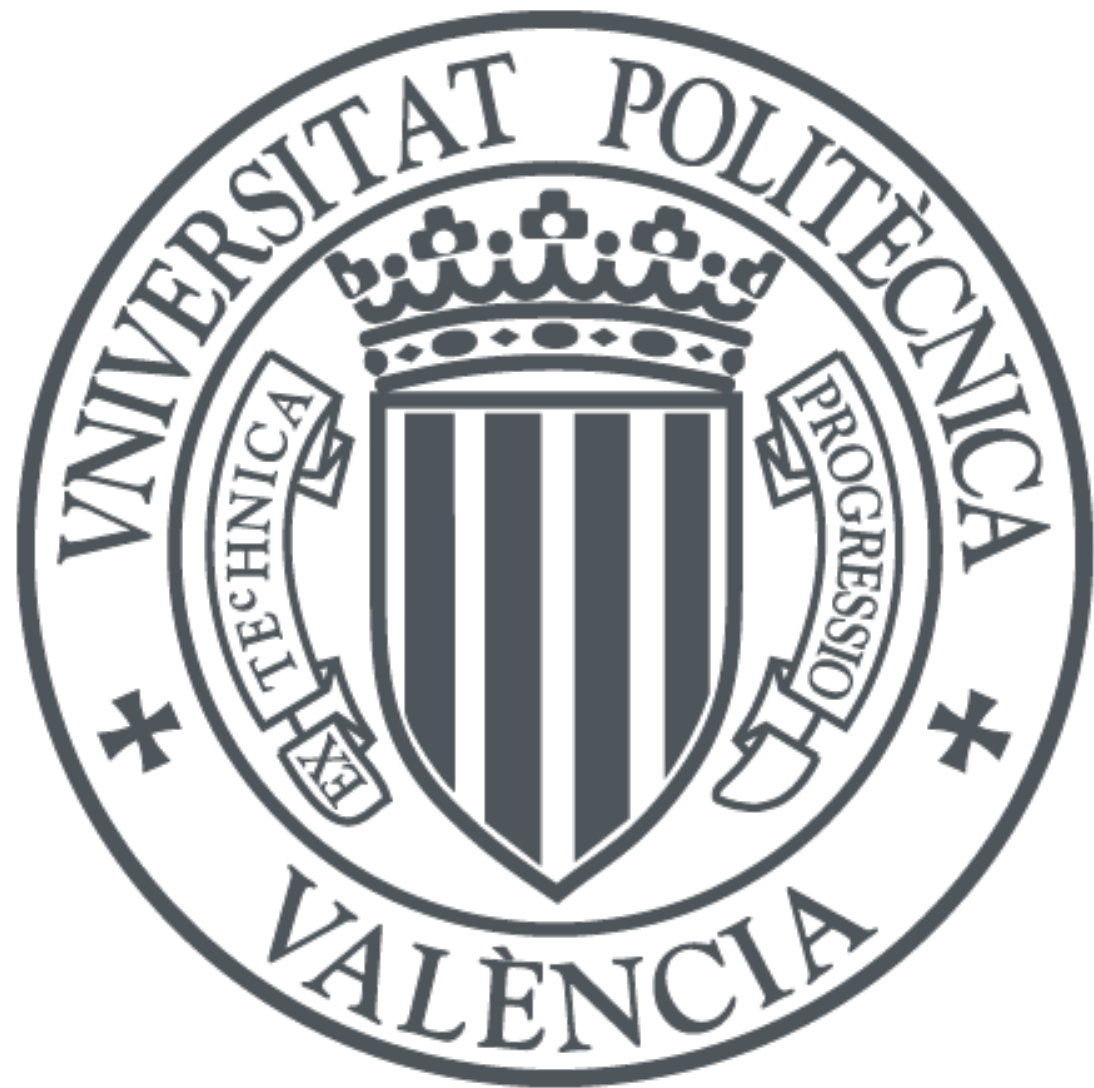

The final publication is available at

http://dx.doi.org/10.1109/TNS.2015.2473695

Copyright Institute of Electrical and Electronics Engineers (IEEE)

Additional Information

"C2015 IEEE. Personal use of this material is permitted. Permission from IEEE must be obtained for all other uses, in any current or future media, including reprinting/republishing this material for advertising or promotional purposes, creating new collective works, for resale or redistribution to servers or lists, or reuse of any copyrighted component of this work in other works." 


\title{
Analysis of the Statistical Moments of the Scintillation Light Distribution With dSiPMs
}

\author{
P. Conde, A. J. González, A. González, L. Hernández, P. Bellido, E. Crespo, A. Iborra, L. Moliner, \\ J. P. Rigla, M. J. Rodrìguez-Álvarez, F. Sánchez, M. Seimetz, A. Soriano, L. F. Vidal, and J. M. Benlloch
}

\begin{abstract}
In $\gamma$-ray detectors, monolithic scintillation crystals offer the possibility of preserving the scintillation light distribution especially when painted black. The statistical moments of this distribution provide accurate information on the three photon impact coordinates, including their depth of interaction (DOI). Digital SiPMs (dSiPMs) return digital information based on pixels about the collected light distribution, since the signal is a digital sum of the trigger bins. In this work we present, for the first time, an accurate analysis of the statistical moments of the light distribution using monolithic painted black crystals and state-of-the-art dSiPMs. Two $32.6 \times 32.6 \mathrm{~mm}^{2}$ monolithic LYSO crystals covering the entire photodetectors area have been used in coincidence with $10 \mathrm{~mm}$ in thickness. The photosensor tiles were kept at a stable temperature of $\mathbf{T}=20^{\circ} \mathrm{C}$. Energy resolution of about $18 \%$ was reached in relation to the zeroth moment. The first moment, related to the impact position, determined a spatial resolution of about $3 \mathrm{~mm}$ near the crystal center, but quadratically degrading tow ards the crystal borders. The DOI resolution, measured by means of the second moment, was found to be nearing $4 \mathrm{~mm}$ in the crystal center region. The third order moment, the so-called skewness, is related to the degree of truncation and once calibrated minimizes the compression effects. A corrected spatial resolution of about $3 \mathrm{~mm}$ was then measured for the entire crystal surface. DOI resolution improved at the crystal's center, reaching $3.5 \mathrm{~mm}$, but a degradation towards the borders remained due to truncation of the scintillation light distribution.
\end{abstract}

Index Terms-Gamma-ray detectors, photodetectors, solid scintillation detectors.

\section{INTRODUCTION}

I N POSITRON emission tomography (PET) the detector blocks are primarily made up of scintillation crystals and photosensors. The $511 \mathrm{keV} \boldsymbol{\gamma}$-photons are commonly converted into scintillation light in order to be photodetected. There are basically two types of crystal configurations used for this purpose, namely pixelated and monolithic.

P. Conde, A. J. González, L. Hernández, P. Bellido, E. Crespo, A. Iborra, L. Moliner, J. P. Rigla, M. J. Rodrìguez-Álvarez, F. Sánchez, M. Seimetz, A. Soriano, L. F. Vidal, and J. M. Benlloch are with the Institute for Instrumentation in Molecular Imaging (I3M), CSIC Universidad Politecnica de Valencia CIEMAT, 46022 Valencia, Spain.

A. Gonzalez is with the Iniversidad of Valencia. Valencia. Snain.
The pixelated crystal configuration is based in an array of scintillator crystals. In this configuration the scintillation light is channeled towards the photosensors with minimal spatial spread. Here, well-defined light distribution is generated at the exit face of the scintillator, typically covering a small number of photosensor units. A linear positioning scheme, usually based on the center of gravity ( $\mathrm{CoG}$ ) algorithm can be used to determine the impacted pixel [1]. The spatial resolution is defined by the crystal pixel size, although light spread and crystal Compton scattering also play an important role. Due to the large number of internal reflections within a single crystal pixel, providing depth of interaction (DOI) information is very complex. The crystal segments can be significantly thicker, which increases the stopping power, while maintaining good spatial resolution. Nearly all commercial scanners use the segmented crystal approach [2]. However, there are a number of drawbacks when compared to the monolithic crystal design. As one aims to improve the spatial resolution, the cross-sectional area of the crystal segments needs to become smaller. This in turn leads to an augment in cost due to the increased complexity of manufacturing smaller crystal segments. The smaller the crystals are made the less light will be collected. Moreover, complicated and expensive detector modifications are necessary to enable DOI detection for pixellated crystals while monolithic crystals inherently can provide information about the DOI.

In detectors based on monolithic scintillators the position needs to be estimated from the light intensity distribution on the photosensor pixels. During the photoconversion process of the incident $\gamma$-ray, the scintillator transforms the deposited energy into visible light that is emitted isotropically from the excited scintillation centers. Since the range of the electrons inside the scintillator will be short, the excited scintillation centers are densely concentrated around this photoconversion position and act similarly to point-like source. Here, it should be noted that the $511 \mathrm{keV}$ photon does not always interact in one unique point inside the crystal. Although a large fraction of photons undergoes Compton scattering in the crystal volume before being totally absorbed, typically about $38 \%$ of the $\gamma$-ray will deposit their energy via photo-electrical absorption at one single point, only few events are characterized by rather long distances between two subsequent Compton and/or photoelectric interactions [3]. The overall contribution of the spread caused by multiple inner crystal scattering to the total scintillation light spread is low for LYSO crystals. The two most distant interactions for a given $\gamma$-ray in the $\mathrm{x}-\mathrm{y}$ plane is about $3 \mathrm{~mm}$. [4]. That being said, some major drawbacks of using monolithic crystals 
need to be noted. More data per event is typically needed, since the light distribution (LD) covers the entire photodetector surface, specially at the scintillator entrance. There is larger influence of dark counts because of larger area required for detecting one event. Monolithic crystals also requires the use of more complex positioning algorithms such as linear algorithms (CoG), statistical algorithms like Least Squares (LS), Chi-Square (Chi2) and Generalised Chi-Square (GenChi2), or more complex methods as the comparison with Nearest Neighbours distributions (kNNs) or supervised learning methods as Neural Networks (NNs) [5]. Simple positioning algorithms $(\mathrm{CoG})$ are based on the preservation of light distribution, so inner reflections need to be avoided, often accomplished by black painting the crystal surfaces, thus reducing the light collection efficiency [6]. Due to the finite size of the scintillator volume, the light distribution is truncated everywhere except for the crystal center. This effect produces a worsening in the spatial response uniformity, especially at the crystal borders (image compression). Consequently, a poor estimation of the interaction positions at the edges is obtained. This situation is especially critical when linear algorithms such as $\mathrm{CoG}$ are used. The statistical methods (LS, Chi2 or GenChi2) could be enabled using a theoretical expression describing the LD. However, the accuracy in the determination of a theoretical expression might be the major limitation to this method. The kNNs method is an extension of the LS method where the input data can be transformed into a reduced representation set of features, thus reducing the size of the reference data set. Nevertheless, the extraction process is the major limitation of this method. The NNs method have been suggested to fit experimental data in a more efficient way when compared to other methods such as LS fitting. The advantage of NNs is the avoidance of iterative processes and that it does not require initialization parameters. However, the training process of the NN is critical in order to obtain an accurate estimation of the LD parameters.

In this work we will study the determination of the 3-D impact coordinates of $511 \mathrm{keV}$ photons with monolithic scintillators through the scintillation light distribution moments, using an array of digital Silicon Photomultipliers (dSiPM) as the photosensor device. A method to minimize the effects of compression using the third moment of scintillation light distribution will be proposed.

\section{Materials AND Methods}

\section{A. Scintillation Light Distribution Model}

The light distribution that is generated inside a monolithic crystal due to $\gamma$-photon interaction can be assumed to spread isotropically [4]. In that way, the amount of visible photons produced in the interaction that reach the surface $\mathrm{d} \mathbf{S}$, in the neighborhood of $\mathbf{r}$, can be described with the inverse square law

$$
J\left(\mathbf{r}, \mathbf{r}_{\mathbf{c}}\right)=\frac{J_{c}}{4 \pi\left|\mathbf{r}-\mathbf{r}_{\mathbf{c}}\right|^{2}} \mathrm{~d} \mathbf{S}, \mathbf{r} \in \mathbb{R}^{3}
$$

where $J_{c}$ is the number of scintillation photons produced at the interaction point, and $\mathbf{r}_{\mathbf{c}}$ are the coordinates of the interaction.

Since the geometry of the entry window of the photonsensors is constrained to the $X Y$ plane, only the optical photons of the

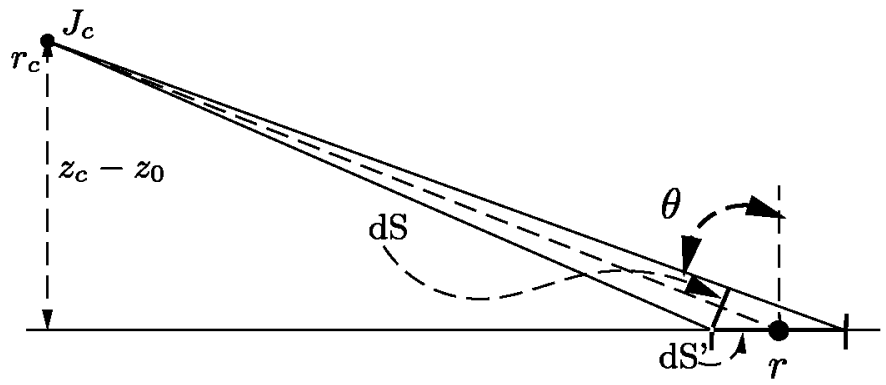

Fig. 1. Diagram of a flat surface element $\boldsymbol{d} \mathbf{S}^{\prime}$ in the sensitive area of the detector, which is irradiated by a source at $\mathbf{r}_{\mathbf{c}}$ and angle $\boldsymbol{\theta}$. A reduction in the density of photons to the factor of $\cos \theta$ is observed since the photon flux $d \mathbf{S}$ is spread over $d \mathbf{S}^{\prime}$.

projection over that plane will be transferred to the photodetectors. Taking into account that the position of the light source is always confined to the scintillator volume, the photons flux corresponding to the surface $d \mathbf{S}$ is covered by the surface $d \mathbf{S}^{\prime}$ of the sensitive area of the photosensors array (see Fig. 1). From this scheme, it can be deduced

$$
d \mathbf{S}=d \mathbf{S}^{\prime} \cos \theta=d \mathbf{S}^{\prime} \frac{z_{c}-z_{0}}{\left|\mathbf{r}-\mathbf{r}_{\mathbf{c}}\right|}
$$

In consequence, the light distribution detected at the $X Y$ plane can be written as

$$
J\left(x^{\prime}, y^{\prime}, \sigma\right)=\frac{J_{c}}{4 \pi} \frac{\sigma}{\left(\sigma^{2}+{x^{\prime}}^{2}+{y^{\prime}}^{2}\right)^{3 / 2}}
$$

where $\sigma=z_{c}-z_{0}$ is the distance from the photodetectors plane to the interaction point and $x^{\prime}=x-x_{c}, y^{\prime}=y-y_{c}$ are the centered coordinates.

\section{B. 3-D Impact Coordinates Estimation}

In position sensitive photodetectors the impact coordinates can be well determined with the first three moments of the sampled distribution, when referring to monolithic crystals [7]

$$
\begin{aligned}
\mu_{n, m} & =\int x^{n} y^{m} J(x, y) d x d y \\
\mu_{n, m}^{\prime}\left(x_{c}, y_{c}\right) & =\int\left(x-x_{c}\right)^{n}\left(y-y_{c}\right)^{m} J(x, y) d x d y
\end{aligned}
$$

Equation (4) shows the two dimensional expression for the statistical moments and equation (5) those for the two dimensional centered moments where $x, y$ are the coordinates for each photodetector, i.e. SiPM. $\boldsymbol{x}_{\boldsymbol{c}}, \boldsymbol{y}_{\boldsymbol{c}}$ are the measured coordinates of the CoG. The zeroth moment $\mu_{0}$ corresponds to the total charge released by a $\boldsymbol{\gamma}$-event which is proportional to the detected photon energy. The first moments $\mu_{1,0}$ and $\mu_{0,1}$ are the mean of the light distribution, providing information about the 2-D ( $\mathrm{x}$ and y) photon impact coordinates. One of the most important centered moment is the variance, $\mu_{2,0}^{\prime}$ and $\mu_{0,2}^{\prime}$, that provides information about the width of the light distribution which are strongly correlate to the DOI. Note that for symmetric distributions, $\mu_{2,0}^{\prime}=\mu_{0,2}^{\prime}$

In monolithic crystals the distribution symmetry is truncated everywhere except for the area near the crystal center due to the finite size of the scintillator. Thus, the estimation of moments 


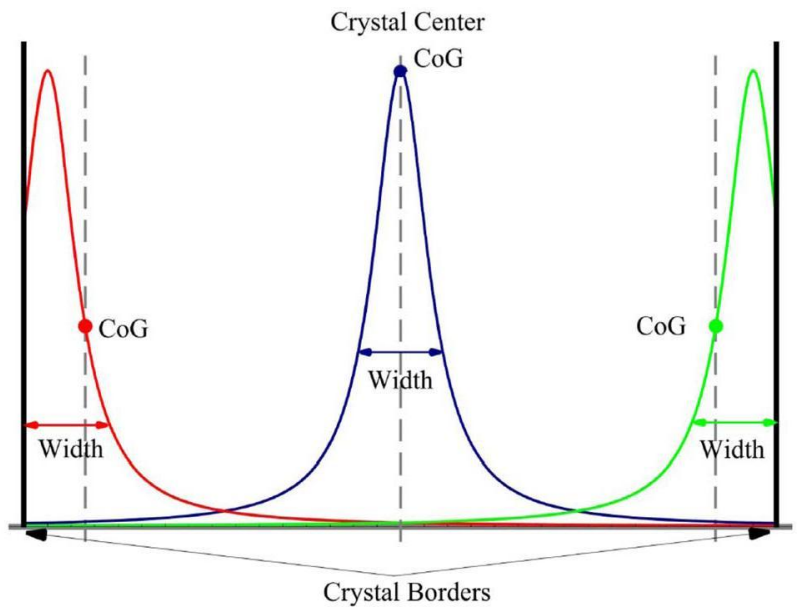

Fig. 2. Ideal light distributions produced at three different impact coordinates. The blue line represents a $\gamma$-event at the scintillator center while the red and green lines are impacts produced near the crystal borders. The first moment (CoG) and second moment (Light distribution width) are poorly estimated when the $\boldsymbol{\gamma}$-event is far from the crystal center.

results in a related error, as depicted in Fig. 2. For the first moment, this error is called compression as it causes the impact position estimation near the edges to be shifted toward the crystal center [8]. This effect can be reduced with the geometry of the crystal, i.e. with a truncated pyramidal geometry [9]. The error in the determination of the centroid also affects the computation of the second centered moment, by resulting in a poor estimation of the DOI for $\gamma$-rays impacting near the crystal borders. The third centered moment, referred to as skewness, provides information about of asymmetry of a distribution and thus corresponding to the degree of truncation of the sampled light distribution. The third moment can be used for further corrections of the impact position estimation obtained with $\mu_{1,0}$ and $\mu_{0,1}$ (CoG algorithm).

\section{C. dSiPMs}

Digital Silicon Photomultipliers (dSiPM), manufactured by Philips Digital Photon Counting, are solid-state single-photon sensitive devices made of arrays of Geiger-mode avalanche photodiodes [10]. In contrast to analog SiPM, these devices integrate CMOS electronics into a silicon photomultiplier chip for early digitization of Geiger-cell output resulting in a fully digital readout [11]. This early digitizing minimizes the electronic readout noise avoiding parasitic capacitances of the on-chip interconnect, the bond wires or the external load. The dSiPM are currently manufactured in two different array configurations, the DPC-6400-22 and the DPC-3200-22. In this work we have used the DPC-3200-22 version. The DPC-3200-22 dSiPM array is composed of $4 \times 4$ individual silicon dice covering an area of $32.6 \times 32.6 \mathrm{~mm}^{2}$. On each die, four dSiPM pixels are arranged in a $2 \times 2$ matrix. This grouping of pixels onto the dice is important since pixels that belong to the same die share a common controller and time-to-digital converter (TDC), i.e. a single timestamp is obtained per die for each event. Each pixel comprises 3200 cells. The discharged cells are summed up on a pixel basis to produce the photon count value. For the purpose of trigger handling, the pixels are further subdivided into quadrants, each of which comprises 800 cells. These four pixel quadrants are referred to as subpixels. Each cell comprises a single photon avalanche diode (SPAD) and additional control circuitry in $180 \mathrm{~nm}$ CMOS technology. This control circuitry facilitates active quenching of the SPAD after breakdown. Similar to analog SiPM, the breakdown of a SPAD can occur due to either photon or thermally generated charge carriers. The cell control circuitry includes functionality for active recharging of the diodes as well as selective enabling/disabling of individual cells [12].

In summary, each DPC-3200-22 dSiPM array has 64 digital outputs providing the number of photons detected on each pixel and 16 outputs containing the timestamp information on a die basis [13].

The acquisition sequence is fully digital. Trigger signals are generated by pixels when the configured trigger threshold is reached on a pixel. Four different trigger schemes are supported, which are based on the different boolean interconnection of the four sub-pixels of a pixel. In this work we use trigger scheme 4, where all sub-pixels are connected by AND-Gates, thus each sub-pixel has to detect a cell breakdown to cause a trigger generation by the pixel. In other words, the use of trigger scheme 4 entails that at least 4 avalanche photodiodes (APDs), one at each sub-pixel, must be fired simultaneously to produce a trigger. After the trigger generation, a validation phase of configurable length (here set to $40 \mathrm{~ns}$ ) starts. The validation network of the digital photon sensors uses, similar to the trigger network, the amount of cell-discharges in considering whether an event is valid or invalid. The rows of a sub-pixel are grouped in row-trigger-lines (RTLs). For the DPC-3200-22 each single row makes up a RTL. Once the RTL of a group detects a cell-discharge within the validation interval, the corresponding input of the connected logic gate is set to logic high level. We used the highest validation threshold, which corresponds to setting all gates as AND, in order to minimize the effect of dark counts. This validation threshold means that one cell in each RTL group of a sub-pixel has to discharge to generate a valid-signal for the corresponding sub-pixel.

As the scintillation light is expected to cover the entire photodetector surface, the dice located far from the scintillation interaction will not pass the threshold and therefore will not be triggered. To avoid this effect, the array is configured to force an event acquisition cycle in all the dice, although most of them will not pass the validation threshold scheme to start their own acquisition sequence. This feature is termed neighbor logic (NL) [14].

A major drawback with the NL is that when the dice which did not provide the primary trigger are in the recharge or holdoff period do not react to the external trigger. Depending on the dark-count rate this can be a considerable effect, significantly reducing the sensitivity of the detector [15].

\section{EXPERIMENTAL SETUP}

Due to the fact that each die contains an individual TDC, the timestamp provided by each of them can be skewed by an offset factor. A skew time calibration between dice before performing measurements is needed [16]. For that purpose we coupled one 


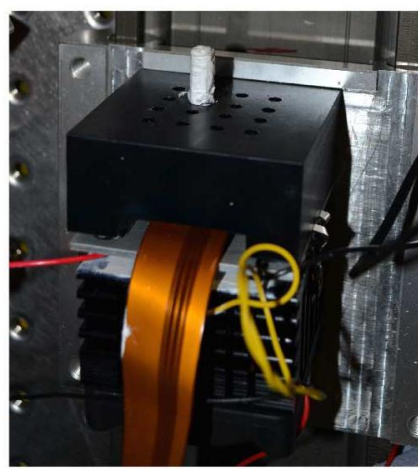

(a)

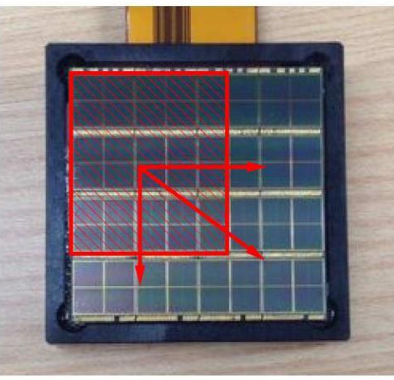

(b)
Fig. 3. (a) Picture of the dSiPM module coupled to a single LYSO crystal (b) Diagram of the pixellated LYSO crystal positions at the dSiPM quadrants.

tile with a single of $3 \times 3 \mathrm{~mm}^{2}$ LYSO crystal covering one die. The other tile was coupled to a pixelated $24 \times 24 \mathrm{~mm}^{2}$ crystal composed of $12 \times 12$ pixels and $2 \mathrm{~mm}$ pitch. The crystal pixels were covered with $70 \mu \mathrm{m}$ of reflective material.

Once the time skew between dice is calibrated, the experimental setup for the rest of measurements is composed of two dSiPMs tiles in coincidence, separated by a fixed distance of $10 \mathrm{~cm}$. Both photosensor modules were coupled with optical grease to a $32.6 \times 32.6 \mathrm{~mm}^{2}$ black-painted (TESTORS Enamel 1149TT, flat black) monolithic LYSO crystals (Proteus, Ohio, US) covering all surfaces except the one coupled and matching the photodetection area. A crystal thickness of $10 \mathrm{~mm}$ was used.

Each detector module was located on a opaque housing. In order to reduce the dark count rate, a custom made Peltier cooling based system was developed with a Peltier module coupled through thermal paste to a heatsink and fan block. Both modules were kept at a stable temperature of $\mathrm{T}=20^{\circ} \mathrm{C}$. A picture of the detector module is shown in Fig. 4(a).

It has been pointed out that the dark count rate can be significantly improved by disabling a relatively small amount of cells, e.g. in the order of $(5-10) \%$ [17]. An inhibition map deactivating $10 \%$ of the cells with higher dark counts was created for each module.

A $30 \mathrm{~mm}$ thick Tungsten block with a drilled hole $1.2 \mathrm{~mm}$ in diameter was placed in between a detector module and a ${ }^{22} \mathrm{Na}$ source $1 \mathrm{~mm}^{3}$ in size to properly collimate the gamma rays. The assembly was sequentially moved along the $\mathrm{X}$ axis [Fig. 4(b)] using a digitally controlled stepper motor. The other detector module was used for coincidence purposes.

\section{EXPERIMENTAL RESULTS}

\section{A. Time Skew Calibration}

The skew time calibration is performed by aligning the mean values of the time difference histograms between the detector with a single LYSO crystal and one selected LYSO crystal per die of the other detector. The pixellated crystal did not cover the whole photodetector area and thus it is necessary four measurements moving the crystal to the photodetectors quadrants [Fig. 3(b)].

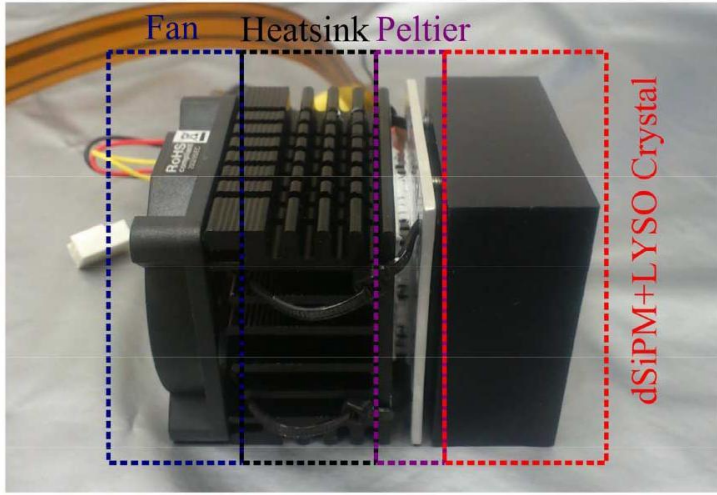

(a)

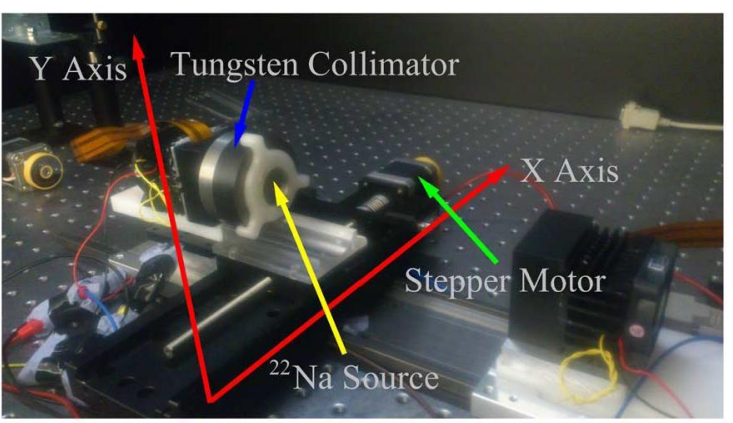

(b)

Fig. 4. (a) Picture of one dSiPM module. The module is composed of a black housing, for light isolation purposes, and a cooling block formed by a Peltier cell, a heatsink and a fan. (b) Picture of the experimental setup. The cartesian axis choice is depicted (red lines).

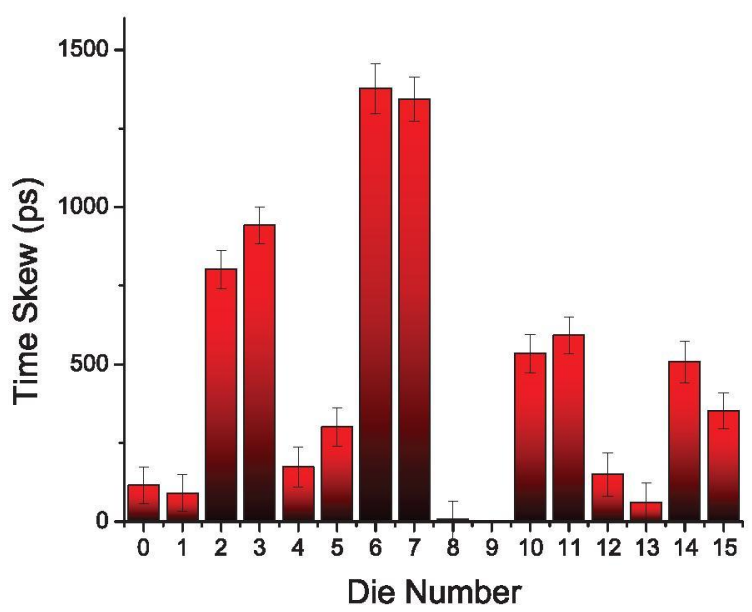

Fig. 5. Time skew differences between dice in one dSiPM module. The error bars are the standard deviation of the measurements.

The skew time calibration showed offset times varying from 7.8 ps to 1378 ps between dice (Fig. 5). Since die 9 was present in all the measurements, it was used as a time origin.

\section{B. Zeroth Moment - Energy Resolution}

The zeroth moment corresponds to the total charge released in the photodetection area. It is measured by adding the number of detected photons for each pixel inside the integration time. 


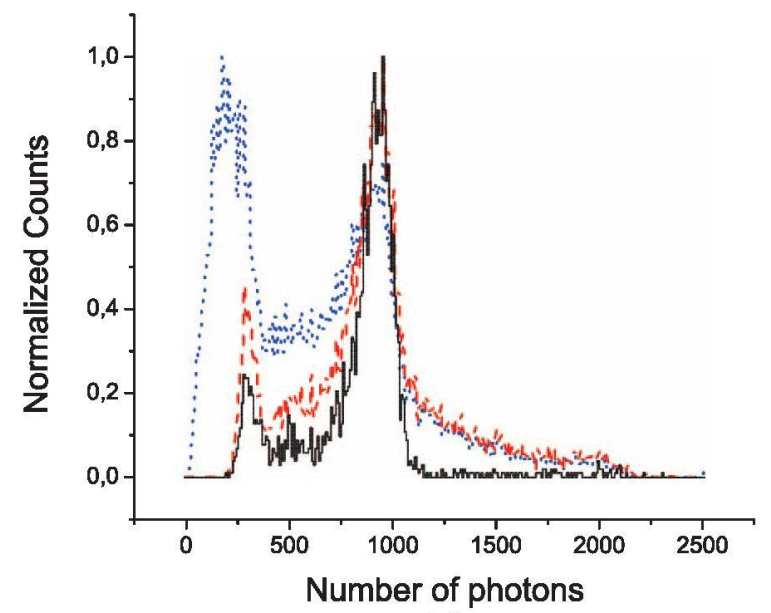

(a)

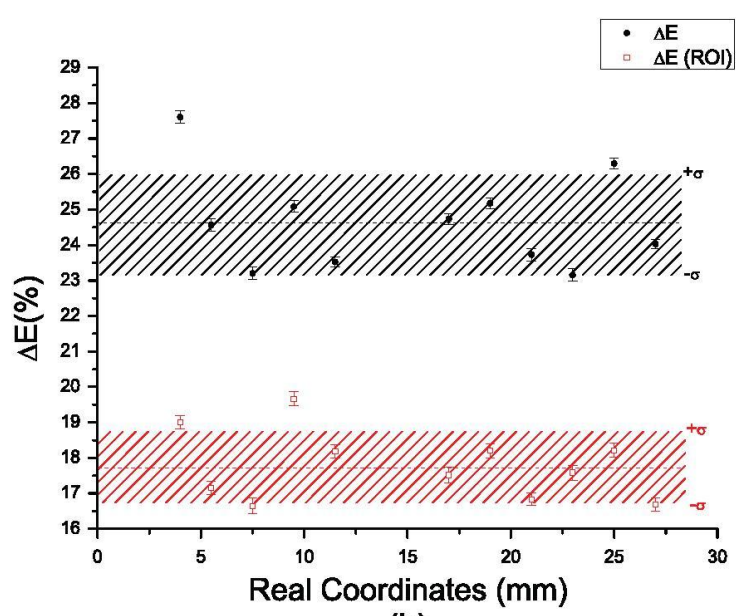

(b)

Fig. 6. (a) The dotted blue lines depicts the energy spectrum considering all the recorded events with NL activated. The dashed red line shows the energy spectrum when the entire photodetector surface is considered and all the dice are triggered. The solid black line is the energy spectrum when events are filtered by a ROI and all the dice are triggered. (b) Plot of the energy resolution obtained at the eleven test source positions, both on the entire crystal surface (black full circles) and at the ROI (red open square). The error bars are the standard error in the centroid determination from the Gaussian fit to the $511 \mathrm{keV}$ photopeaks.

Since the NL was activated, we expected to have photon counts from all the dice in the dSiPMs tiles for each event. However, as was mentioned in Section II, some dice can be in the recharge or holdoff period during an integration process, so the photon counts of those dice are missing. Fig. 6(a) depicts the difference between the recorded energy spectrum considering either all the events (blue line) or events where all the dice are triggered (red line). It can be seen that the low energy peak, mainly due to dark count contribution, is significantly reduced for a collection of valid events. Moreover, the overall spectrum is improved. The fraction of valid events is related to the dSiPMs temperature. In this work, at $\mathbf{T}=(20 \pm 1)^{\circ} \mathrm{C}$, valid events represent a fraction of $(39 \pm 2) \%$ of the total events in coincidence mode.

At each measured position, we calculated the energy resolution for the entire photodetector area and the energy resolution in a region of interest (ROI) around the source position [Fig. 6(b)]. A mean energy resolution of $(24.6 \pm 1.4) \%$ and

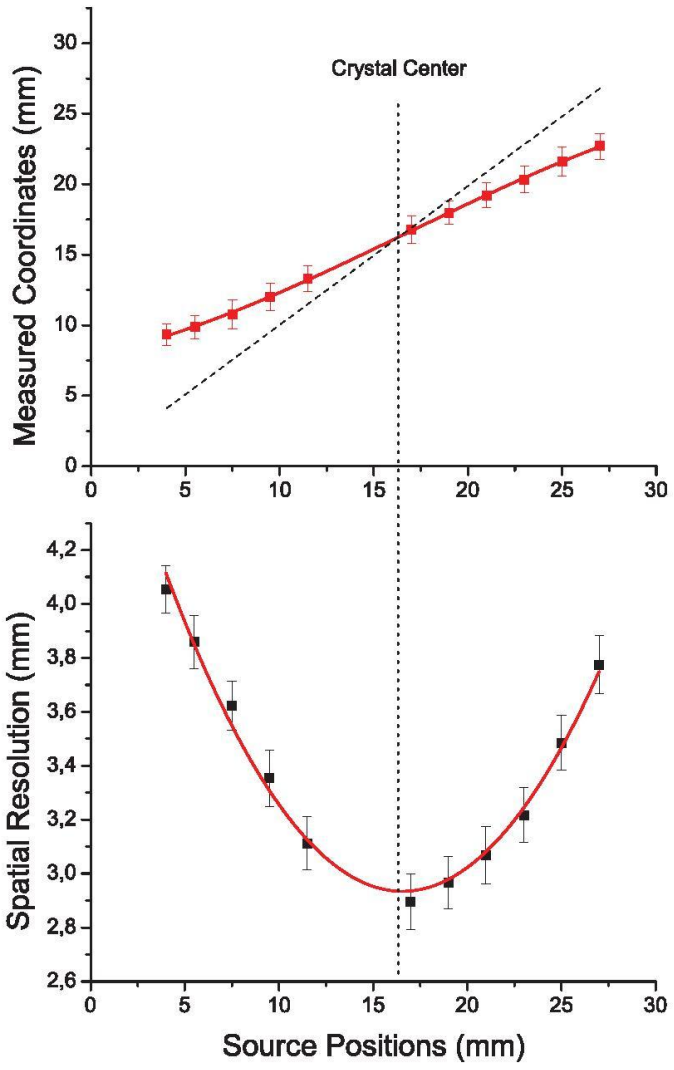

Fig. 7. Top: Plot of the impact coordinates obtained using the $\mathrm{CoG}$ (red points). The CoG points are fitted to a third order polynomial (red line). The dashed black line represents the linearity between the real and the measured positions. Bottom: Plot of results for the spatial resolution (FWHM) along one detector axis using the CoG method. The solid red line is a second order polynomial fit to the values. The error bars are the standard error in the centroid determination from the Gaussian fit to the first moment distributions.

$(17.6 \pm 0.9) \%$ is found on average for the entire detector and ROI, respectively.

\section{First Moment - Spatial Resolution}

The histograms of the first moment for the collimated ${ }^{22} \mathrm{Na}$ source along one axis were fitted to a Gaussian distribution, for which the mean value was taken as the impact coordinates of the $\gamma$-interaction. As depicted in Fig. 7, the linearity between the real impact coordinates and measured coordinates, when these are determined using the CoG algorithm, is lost except for a small region near the crystal center. The spatial resolution is calculated as the FWHM of the histograms obtained after correcting the measured CoG values with the source locations, using the third order polynomial fit [18] (Fig. 7, top). This procedure had been successfully applied by the authors to gamma ray detection devices based on continuous scintillators [19]-[21]. Fig. 7 shows a spatial resolution of $(2.9 \pm 0.1) \mathrm{mm}$ near the crystal center. The spatial resolution quadratically degrades towards the crystal borders. A spatial resolution of $(4.0 \pm 0.1) \mathrm{mm}$ is found near the edges.

\section{Second Moment - DOI Resolution}

The correlation between the DOI and the second centered moment of the scintillation light distribution in monolithic crystals is a direct consequence of the inverse square law. 


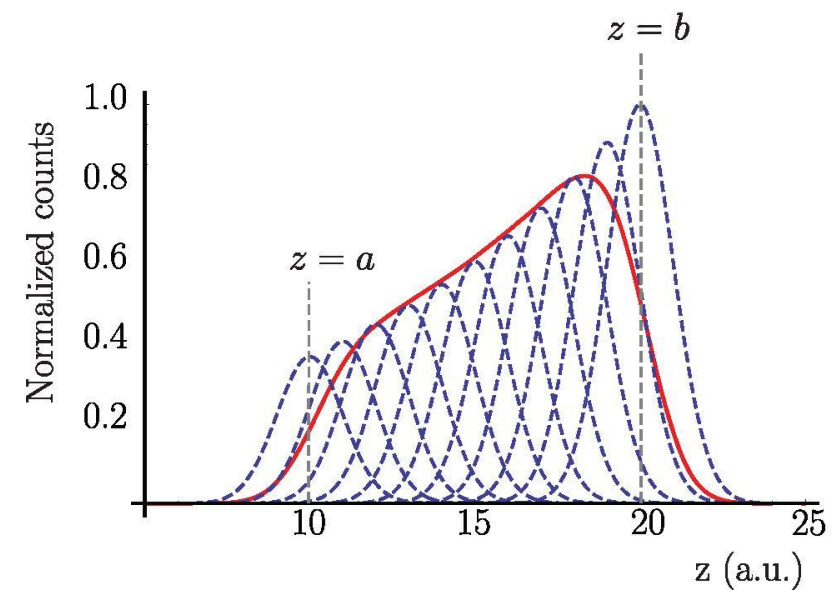

Fig. 8. Theoretical distribution of DOIs for a $\boldsymbol{\gamma}$-ray beam impinging perpendicular to the photodetector array. Parameters a, b represents the crystal limits. The dashed blue lines are the theoretical DOI distributions at different depths and the solid red line represents the analytical DOI distribution.

For a collimated $\boldsymbol{\gamma}$-ray beam impinging normal on the detector, the measured DOIs are distributed following an exponentially decaying distribution. Therefore, the distribution of the measured second order moments is expected to be a superposition of the detector intrinsic DOI distributions at different depths, weighted by a decreasing exponential due to the $\boldsymbol{\gamma}$-ray attenuation [Fig. 8(a)].

Making the assumption that the intrinsic DOI resolution is constant along the crystal depth, we can define an analytical expression for the DOIs distribution [22]

$$
D O I(z)=A e^{-\alpha z}\left[\operatorname{Erf}\left(\frac{b-z}{\sqrt{2} \sigma_{i n t}}\right)-\operatorname{Erf}\left(\frac{a-z}{\sqrt{2} \sigma_{i n t}}\right)\right]
$$

where $\alpha$ is the linear attenuation coefficient of the crystal and remains constant. The fitting parameters are $A$ which is a normalization parameter, $\boldsymbol{a}, \boldsymbol{b}$ which are the limits of the scintillator thickness and $\sigma_{i n t}$ that is the intrinsic DOI resolution. In Fig. 8(b) we show the experimental DOI distribution we have measured at the center of the crystal and the fitted function using equation (6). For impact coordinates near crystal borders, the equation (6) approaches a Gaussian distribution. At this limit, the parameters $\mathrm{a}$ and $\mathrm{b}$ are too close to each other for a sensible prediction of the DOI resolution.

Fig. 8(c) shows a DOI resolution $(\triangle D O I)$ degradation towards the crystal borders when measured using the second centered moment. This is a consequence of the truncation of light distribution. At the crystal center $\triangle D O I$ is $(4.3 \pm 0.1) \mathrm{mm}$ and at the crystal borders it is about $(7.1 \pm 0.4) \mathrm{mm}$.

\section{E. Third Moment - Skewness Correction}

The third centered moment, namely skewness, can be used as a measure of the degree of truncation of the sampled light distribution for each event. Since our measurements were only carried out along one axis, we only measure the third moment related to that axis $\left(\mu_{3,0}\right)$. It should be noted that the skewness value is correlated to the DOI, but for simplicity we assume a mean $z$ value for all the measures.

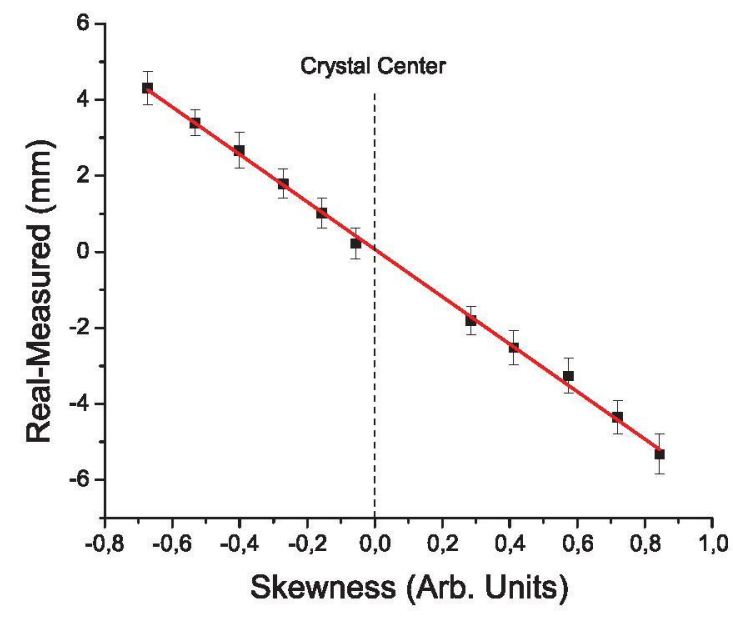

Fig. 9. Plot of the mean values of the third moment. The red line depicts a first order polynomial fit to the values. The error bars are the standard error in the centroid determination from the gaussian fit of the third moment distributions.
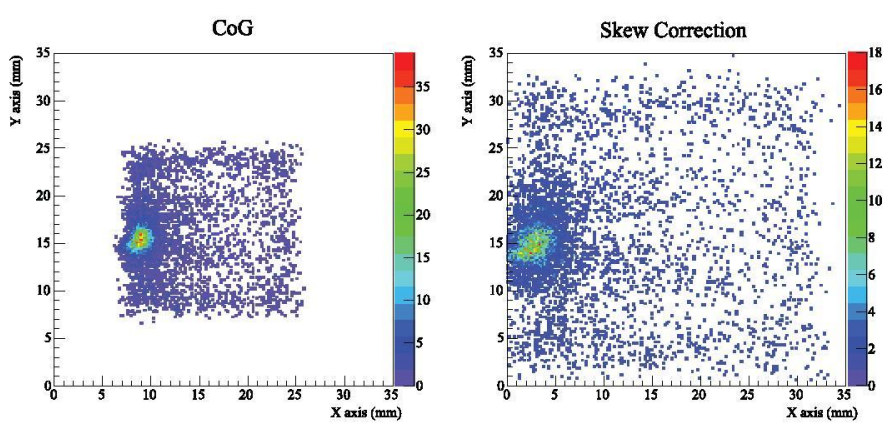

Fig. 10. Left: Density plot of $\gamma$-events with the collimated ${ }^{22} \mathrm{Na}$ source near one crystal border obtained through the first moment (CoG). Right: Density plot after skewness correction.

For calibration purposes, we fit the values of the skewness distribution measured at a given source position to a Gaussian distribution. The skewness coefficient at that calibration point is then determined as the centroid of the fitted Gaussian function. This procedure is repeated for all source positions considered in the calibration process. Once the skewness coefficient is calculated for all these positions, the measured position should be corrected by considering the value of this coefficient. We fitted the difference between the real positions and those measured to a first order polynomial by least squares

$$
x_{\text {source }}^{i}-x_{\text {measured }}^{i}=A_{1} S^{i}
$$

where $i$ varies between 1 and the number of source positions considered in the experiment, $A_{1}$ is the fitted parameter and $S$ $=\mu_{3,0}$. The fit to the calibration data is shown in Fig. 9. A value of $r^{2}=0.9985$ was obtained for the fitted data.

With the fitted parameter, $A_{1}$, and using equation (7), we correct the center estimation of every event. Fig. 10 shows the resulting density plot for CoG and includes the skewness correction.

A mean spatial resolution of $(3.0 \pm 0.1) \mathrm{mm}$ for the entire crystal surface is measured when the impact coordinates are corrected by means of the third moment. In Fig. 11 (top) we have plotted the corrected spatial resolution, observing a linear trend 


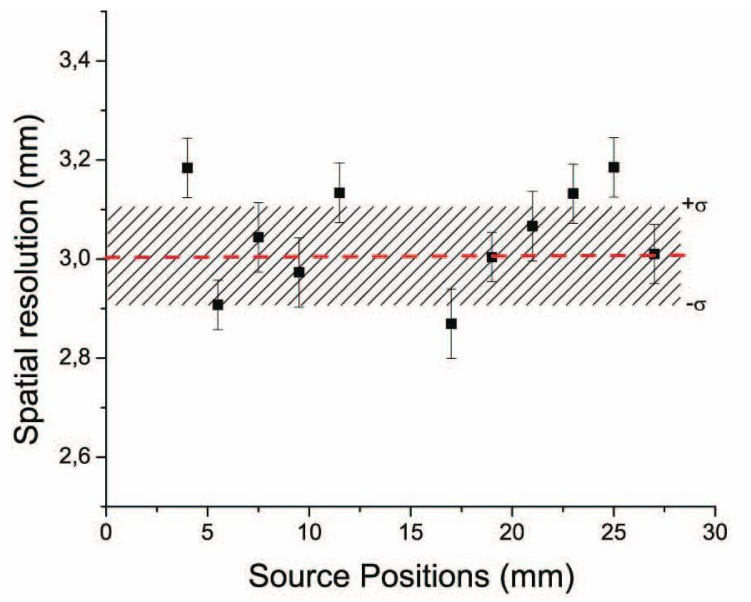

(a)

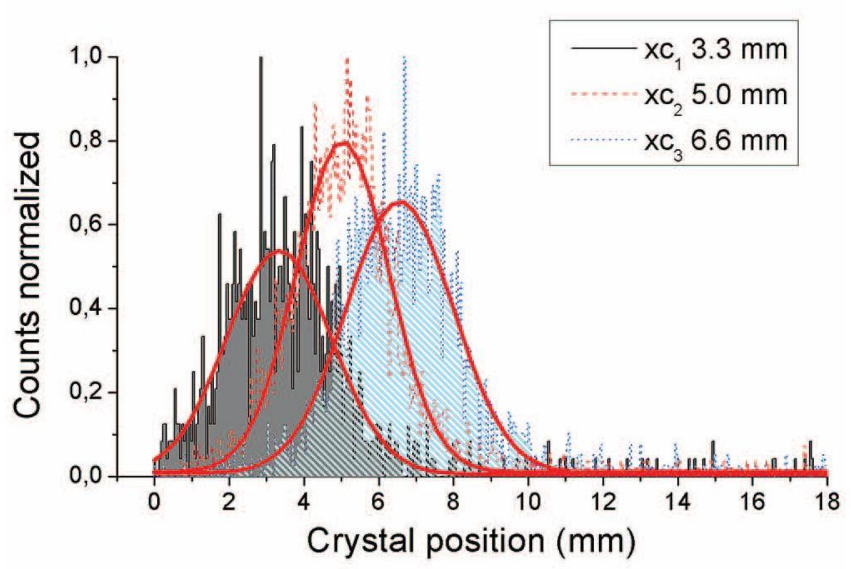

(b)

Fig. 11. Top: Plot of the spatial resolution along one detector axis after correction with the third moment. The red dashed line shows the mean value. Bottom: Three source profiles and fitted curves near the crystal edge.

of FWHM along the X-axis. This figure also shows (bottom) the profiles of three distributions near the crystal edge. The fitted curves (red lines) to the experimental data are also depicted. Using the coordinates of the corrected center, we further recalculated the second centered moment (see Fig. 12). As in the computation of the second moment, the impact coordinates near to the crystal borders showed $a$ and $b$ parameters too close to each other for a sensible prediction of the DOI resolution. The comparison of DOI with CoG and with skewness correction shows a slightly improved for the latter, especially near the crystal center region.

\section{CONClusions AND OutLook}

In this work we have shown the capabilities of determining 3-D photon impact coordinates with monolithic scintillators by determining the first three statistical moments. The second moment is used to determine the DOI, while the third moment is used to correct the marked non-linearity observed in monolithic scintillator crystals, especially toward the crystal edges.

An average energy resolution of $18 \%$ is reached, with blackpainted monolithic crystals coupled to digital SiPMs, at a stable temperature of $\mathbf{T}=20^{\circ} \mathrm{C}$. A spatial resolution (FWHM) of about $3 \mathrm{~mm}$ at the crystal center has been measured using the

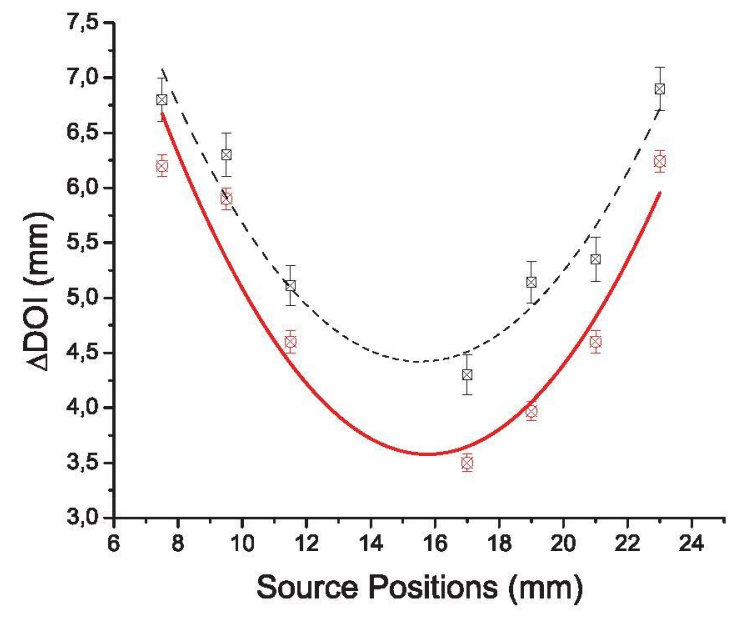

Fig. 12. Plot of the DOI resolution along one detector axis before (black square) and after (red circle) applying the skewness correction. The solid and dashed lines are a second order polynomial fit to the values respectivily.

CoG algorithm. Due to border effects, the spatial resolution degrades quadratically towards the crystal borders. Calibrating the third moment of the measured light distribution minimized the border effects. Spatial resolution of about $3 \mathrm{~mm}$ for the entire crystal area is obtained using this method (see Fig. 11). Using the second moment of the scintillation light distribution, we measured a DOI resolution of $4.3 \mathrm{~mm}$ at the crystal center. When the correction of the third moment is applied to the centroids estimation, the DOI resolution improves to $3.5 \mathrm{~mm}$.

These novel results have shown the capability of using monolithic block crystals in combination with digital SiPM, for innovative cost-effective PET systems developments. Since the NL is mandatory when monolithic crystals are used, the detector sensitivity is reduced near room temperature. However, further studies lowering the detector temperature are needed aiming to improve the detector sensitivity.

Due to the relatively small crystal thickness $(10 \mathrm{~mm})$ we have not been able to properly determine the DOI resolution for impacts far from the crystal center coordinates. Our current aim is to extend these studies to the use of non-black painted monolithic crystals in order to increase the light collection, as well as thicker scintillator crystals in order to obtain better DOI resolutions.

\section{REFERENCES}

[1] S. Siegel, R. Silverman, Y. Shao, and S. Cherry, "Simple charge division readouts for imaging scintillator arrays using a multi-channel pmt," IEEE Trans. Nucl. Sci., vol. 43, no. 3, pp. 1634-1641, Jun. 1996.

[2] B. J. Pichler, H. F. Wehrl, and M. S. Judenhofer, "Latest advances in molecular imaging instrumentation," J. Nucl. Med., vol. 49, pp. 5S-23S, Jul. 2008, Suppl. 2.

[3] C. Lerche, "Design studies for a new $\boldsymbol{\gamma}$-ray detector with depth of interaction ability," M.S. thesis, Univ. Valencia, Valencia, Spain, 2003.

[4] C. Lerche, J. Benlloch, F. Sanchez, N. Pavon, B. Escat, E. Gimenez, M. Fernandez, I. Torres, M. Gimenez, A. Sebastia, and J. Martinez, "Depth of gamma;-ray interaction within continuous crystals from the width of its scintillation light-distribution," IEEE Trans. Nucl. Sci., vol. 52, no. 3 , pp. 560-572, Jun. 2005.

[5] P. G. de Acilu, I. Sarasola, M. Canadas, R. Cuerdo, P. R. Mendes, L. Romero, and C. Willmott, "Study and optimization of positioning algorithms for monolithic pet detectors blocks," J. Instrumentation, vol. 7, no. 06, p. C06010, 2012 [Online]. Available: http://stacks.iop.org/ $1748-0221 / 7 / \mathrm{i}=06 / \mathrm{a}=\mathrm{C} 06010$ 
[6] C. W. Lerche, A. Ros, V. Herrero, R. Esteve, J. M. Monzo, Á. Sebastia, F. Sanchez, A. Munar, and J. M. Benlloch, "Dependency of energy, position and depth of interaction resolution on scintillation crystal coating and geometry," IEEE Trans. Nucl. Sci., vol. 55, no. 3, pp. 1344-1351, Jun. 2008

[7] P. Conde, A. J. Gonzalez, L. Hernandez, L. Moliner, A. Orero, M. J. Rodriguez, F. Sanchez, A. Soriano, L. F. Vidal, and J. M. Benlloch, "First results of an ASIC controlled $\boldsymbol{\gamma}$-detector based on a SiPM-array and a monolithic LYSO," in Proc. IEEE Nuclear Science Symp. Medical Imaging Conf. Rec. (NSSMIC), Oct. 2012, pp. 412-414.

[8] P. Conde, A. González, L. Hernández, P. Bellido, A. Iborra, E. Crespo, L. Moliner, J. Rigla, M. Rodrìguez-Alvarez, F. Sánchez, M. Seimetz, A. Soriano, L. Vidal, and J. Benlloch, "Results of a combined monolithic crystal and an array of ASICs controlled SiPMs," Nucl. Instrum. Meth. Phys. Res. A, vol. 734, pp. 132-136, 2014.

[9] A. Ros, C. W. Lerche, A. Munar, F. Sanchez, A. Sebastia, and J. Benlloch, "Impact of crystal quality, geometry and surface finish for 3D impact position measurements in gamma ray detection systems," in Proc. IEEE Nuclear Science Symp. Conf. Rec., vol. 6, pp. 4246-4251.

[10] C. Degenhardt, G. Prescher, T. Frach, A. Thon, R. de Gruyter, A. Schmitz, and R. Ballizany, "The digital Silicon Photomultiplier; A novel sensor for the detection of scintillation light," in Proc. $\mathrm{Nu}$ clear Science Symp. Medical Imaging Conf. (NSS/MIC), 2009, pp. 2383-2386.

[11] T. Frach, G. Prescher, C. Degenhardt, and B. Zwaans, "The digital silicon photomultiplier; System architecture and performance evaluation," in Proc. Nuclear Science Symp. Medical Imaging Conf. (NSS/ MIC), 2010, pp. 1722-1727.

[12] Y. Haemisch, T. Frach, C. Degenhardt, and A. Thon, "Fully digital arrays of silicon photomultipliers (dSiPM) a scalable alternative to vacuum photomultiplier tubes (PMT),"Phys. Procedia, vol. 37, no. 0 , pp. 1546-1560, 2012.

[13] C. Degenhardt, B. Zwaans, T. Frach, and R. de Gruyter, "Arrays of digital silicon photomultipliers; Intrinsic performance and application to scintillator readout," in Proc. Nuclear Science Symp. Medical Imaging Conf. (NSS/MIC), 2010, pp. 1954-1956.

[14] D. Schug, P. Duppenbecker, P. Gebhardt, B. Weissler, B. Zwaans, F. Kiessling, and V. Schulz, "First evaluations of the neighbor logic of the digital SiPM tile," in Proc. Nuclear Science Symp. Medical Imaging Conf. (NSS/MIC), 2012, pp. 2817-2819.
[15] R. Marcinkowski, S. Espana, R. Van Holen, and S. Vandenberghe, "Effects of dark counts on digital silicon photomultipliers performance," in Proc. Nuclear Science Symp. Medical Imaging Conf. (NSSMIC), Oct. 2013, pp. 1-6.

[16] R. Marcinkowski, S. Espana, H. Thoen, and S. Vandenberghe, "Performance of digital silicon photomultipliers for time of flight PET scanners," in Proc. Nuclear Science Symp. Medical Imaging Conf. (NSS MIC), 2012, pp. 2825-2829.

[17] M. Radoslaw, S. Espana Palomares, R. Van Holen, and S. Vandenberghe, "Effects of dark counts on digital silicon photomultipliers performance," in Nuclear Science Symp. Medical Imaging Conf., p. 6.

[18] J. Benlloch, V. Carrilero, A. González, J. Catret, C. Lerche, D. Abellán, F. G. de Quirós, M. Giménez, J. Modia, F. Sánchez, N. Pavón, A. Ros, J. Martìnez, and A. Sebastiá "Scanner calibration of a small animal PET camera based on continuous LSO crystals and flat pane PSPMTs," Nucl. Instrum. Meth. Phys. Res. A, vol. 571, no. 12, pp. 26-29, Feb. 2007.

[19] F. Sanchez, L. Moliner, C. Correcher, A. González, A. Orero, M Carles, A. Soriano, M. Rodriguez-Alvarez, L. A. Medina, F. Mora, and J. Benlloch, "Small animal PET scanner based on monolithic LYSO crystals: Performance evaluation," Med. Phys., vol. 39 , no. 2 pp. 643-653, Feb. 2012

[20] L. Moliner, A. González, A. Soriano, F. Sanchez, C. Correcher, A. Orero, M. Carles, L. Vidal, J. Barbera, L. Caballero, M. Seimetz, C. Vazquez, and J. Benlloch, "Design and evaluation of the MAMMI dedicated breast PET," Med. Phys., vol. 39, no. 9, pp. 5393-5404, Sep. 2012.

[21] F. Sánchez, A. Orero, A. Soriano, C. Correcher, P. Conde, A. González, L. Hernández, L. Moliner, M. J. Rodrguez-Alvarez, L. F. Vidal, J. Benlloch, S. E. Chapman, and W. M. Leevy, "ALBIRA: A small animal PET/SPECT/CT imaging system," Med. Phys., vol. 40, no. 5, p. 051906, Apr. 2013

[22] C. W. Lerche, M. Döring, A. Ros, V. Herrero, R. Gadea, R. J. Aliaga, R. Colom, F. Mateó, J. M. Monzó, N. Ferrando, J. F. Toledo, J. D. Martìnez, A. Sebastiá, F. Sanchez, and J. M. Benlloch, "Depth of interaction detection for $\boldsymbol{\gamma}$-ray imaging," Nucl. Instrum. Meth. Phys. Res. $A$, vol. 600, pp. 624-634, Mar. 2009. 